\section{Acquired hemophilia A presenting in an elderly man}

$\infty \quad$ See related article page 339

The case: An 85-year-old man presented with bruising of his hands, which he first noticed after shovelling snow 3 days earlier. His past medical history included benign prostatic hypertrophy and glaucoma. He was not taking anticoagulants or nonsteroidal anti-inflammatory drugs. Over the last 3 days the bruising had become extensive, encompassing the palmar and dorsal aspect of both hands and spreading to the lower forearms (Figure 1). The patient had no other bruising, nosebleeds, hematuria, bloody stools or hemoptysis, and he reported having no joint or muscle pains. He did not have a history of liver disease, nor did he have any personal or family history of bleeding or clotting disorders.

On examination, the patient looked well and was afebrile, and his vital signs were stable. Aside from the extensive bruising of both hands, no other bruising, petechiae or sites of active bleeding were discovered, and there was no evidence of hemarthrosis.

Initial laboratory investigations revealed a normal complete blood count, with a hemoglobin level of $130 \mathrm{~g} / \mathrm{L}$, a platelet count of $199 \times 10^{\circ} / \mathrm{L}$ and normal electrolyte levels. Kidney and liver function were normal, as was the blood glucose level. The D-dimer level was slightly elevated, at 250-500 (normal
< 250) $\mathrm{ng} / \mathrm{mL}$. The prothrombin time and international normalized ratio were normal; however, the activated partial thromboplastin time was elevated, at 117 (normal < 35) seconds. A 1:1 mixing assay initially showed a corrected activated partial thromboplastin time of 41 seconds; however, a timedelayed 1:1 mix could not correct the thromboplastin time, which suggested that clotting factor inhibitors were present in the patient's blood. Because only the activated partial thromboplastin time was affected, we assayed for clotting factors specific to the intrinsic pathway and determined that the patient had a factor VIII deficiency (titre $<0.01$ [normal 0.5-1.5] U/mL). Further assays revealed factor VIII inhibitors in the patient's serum, at a level of 12.0 (normal o) Bethesda units. Acquired hemophilia was diagnosed.

The patient was admitted to hospital and given oral prednisone therapy (6o mg/d). Because no sites of active bleeding were identified, no additional treatment was initiated. His activated partial thromboplastin time gradually improved, and no further bleeding or bruising occurred. He was discharged home 4 days later and given a tapered course of prednisone.

One month after the patient completed the course of prednisone, his ac- tivated partial thromboplastin time was again prolonged. He required a combined course of cyclophosphamide and prednisone. His condition is currently maintained on $50 \mathrm{mg}$ of cyclophosphamide daily, with a normal activated partial thromboplastin time and no further bleeding.

Investigations into the cause of this patient's acquired hemophilia included chest radiograph, computed tomography of the chest and abdomen, and blood work to rule out malignant or autoimmune diseases. Findings were normal, and the acquired hemophilia was assumed to be idiopathic in nature.

Acquired hemophilia $\mathrm{A}$ is rare, with an estimated annual incidence of I per million people. This potentially lifethreatening hemorrhagic disorder is caused by the development of autoantibodies to factor VIII of the coagulation cascade. Its distribution is bimodal, with the first peak occurring among young women in the postpartum period and the second among elderly patients, in whom it is frequently associated with autoimmune disease, malignant disease and allergic drug reactions (Box I).

The pattern of bleeding in acquired hemophilia differs from that in congenital hemophilia: bleeding tends to occur in soft tissue, muscle, the retroperitoneal space and the gastrointestinal and genitourinary tracts. Unlike in congenital hemophilia, hemarthrosis is rare. Along with severe bleeding, patients may experience complications such as compart-

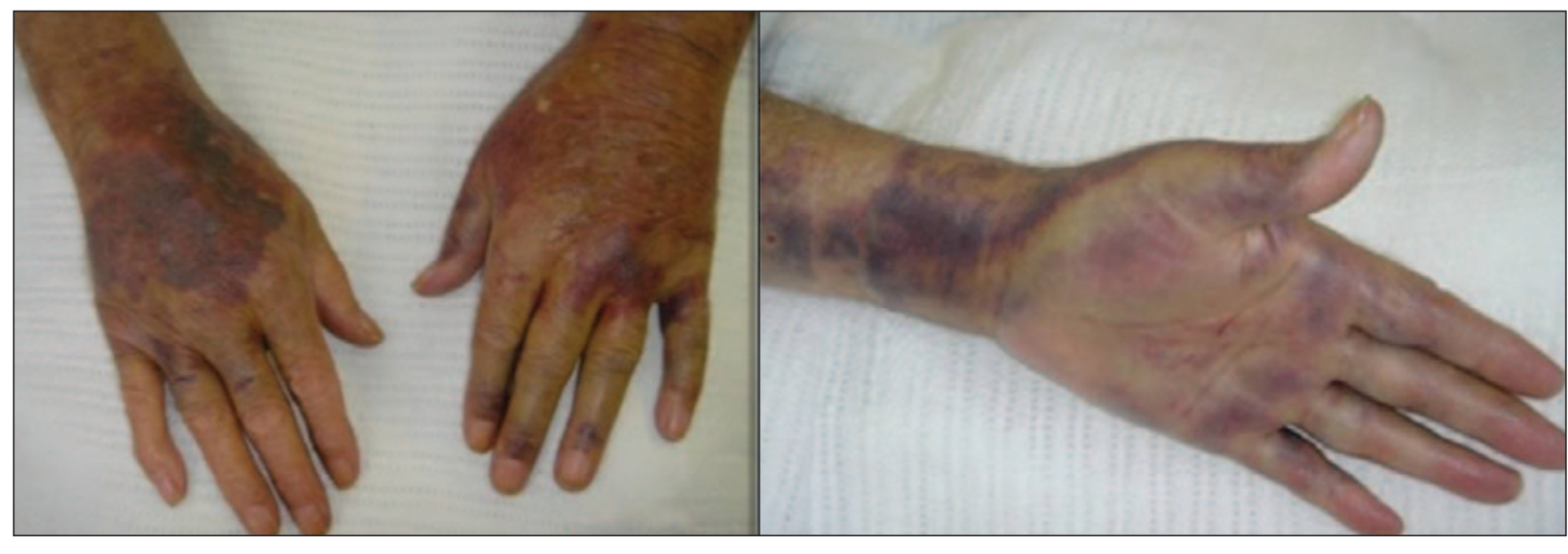

Figure 1: Hands of elderly man showing bruising on palmar and dorsal aspects of both hands that extended to forearms. 
Box 1: Conditions associated with acquired hemophilia

- Idiopathy

- Pregnancy or postpartum period

- Autoimmune disorder (lupus erythematosus [most common], Graves' disease, multiple sclerosis, myasthenia gravis, rheumatoid arthritis, Sjögren syndrome)

- Inflammatory bowel disease

- Diabetes

- Dermatologic disorders (psoriasis and pemphigus vulgaris)

- Respiratory diseases (asthma, chronic obstructive pulmonary disease)

- Allergic reaction to drug (penicillin, sulfonamide, phenytoin, methyldopa)

- Acute hepatitis B or C

- Malignant disease (solid tumour or hematologic)

ment syndrome and hemorrhagic stroke. The rate of death from acquired hemophilia has been estimated at $8 \%-22 \%$, with most deaths occurring in the first few weeks after presentation. ${ }^{1}$

Diagnosis can be challenging, since the patient will have no personal or family history of bleeding disorders. Among elderly patients, warfarin therapy may lead to incorrect evaluation of coagulation studies, and many elderly patients also report superficial bruising and bleeding while taking acetylsalicylic acid therapy. However, in any patient who presents with new-onset severe or deeptissue bleeding, acquired hemophilia should be considered. The diagnosis of acquired hemophilia is based on a prolonged activated partial thromboplastin time, which may be corrected initially by mixing the patient's plasma with an equal volume of normal plasma. However, after prolonged pre-incubation of the plasma, the activated partial thromboplastin time increases again as the inhibitors present in the patient's plasma prevent proper function of the coagulation factors in the normal plasma. ${ }^{2}$ Quantitative assays may reveal a reduced level of factor VIII, and laboratories can directly detect the presence of factor VIII inhibitors in the serum using the Bethesda assay, where I Bethesda unit is

\section{Box 2: Management options for acquired hemophilia A}

\section{Control acute bleeding}

- Hemodynamic stabilization

- Transfusion of packed red blood cells, fresh frozen plasma or cryoprecipitate as necessary

- If the factor VIII inhibitor titre is low (< 5 Bethesda units), consider use of human factor VIII or desmopressin

- If the factor VIII inhibitor titre is $>5$ Bethesda units, consider use of prothrombin complex, porcine factor VIII or recombinant activated factor VIla

- If bleeding is severe and the inhibitor titre is high, consider use of plasmapheresis, intravenous immunoglobulin or immunoadsorption using staphylococcal protein A

Immunosuppressive therapy

- Prednisone

- Consider adding cyclophosphamide (or azathioprine in women of child-bearing age)

- Consider interferon- $\alpha$ or rituximab

defined as the quantity of inhibitor that neutralizes $50 \%$ of clotting factor activity in normal plasma. ${ }^{3}$

Therapy for acquired hemophilia focuses on 2 goals: control of acute bleeding and chronic immunosuppressive therapy to prevent further development of factor VIII inhibitors (Box 2).

Acute bleeding should be treated with hemodynamic stabilization, including transfusions of packed red blood cells, fresh frozen plasma or cryoprecipitate as necessary. Patients with a low titre of inhibitor $(<5$ Bethesda units) can also initially be treated with human factor VIII or desmopressin, ${ }^{4}$ and those with a higher titre may respond to prothrombin complex, porcine factor VIII or recombinant activated factor VIIa. ${ }^{5}$ In patients with severe bleeding and high inhibitor titres, therapeutic plasmapheresis, intravenous immunoglobulin or immunoadsorption using staphylococcal protein A may be considered. ${ }^{1}$

Immunosuppresive therapy should begin immediately once acquired hemophilia is diagnosed. Prednisone will lead to eradication of autoantibodies in $30 \%$ of patients; this proportion increases to $50 \%-70 \%$ when the drug is given in combination with cyclophosphamide. Because cyclophosphamide may cause infertility, azathioprine should be used instead in women of child-bearing age. ${ }^{2}$ Recent studies have shown success with the use of biological agents such as interferon- $\alpha$ and rituximab. In patients whose acquired hemophilia is due to malignant disease, treatment of the underlying disease often eradicates the coagulation autoantibodies. ${ }^{1}$

Although rare, acquired hemophilia can result in serious, life-threatening bleeding. Initial hemodynamic stabilization followed by treatment with immunosuppressive therapy is straightforward and highly successful. The diagnosis of acquired hemophilia should be considered in any patient who presents with bleeding and a prolonged activated partial thromboplastin time.

\section{Stacy Woods MD MSc}

Northern Ontario School of Medicine Northeastern Ontario Family Medicine Laurentian University

Boji Varghese MBBS MD

Department of Endocrinology

Sudbury Regional Hospital

Sudbury, Ont.

This article has been peer reviewed.

Competing interests: None declared.

\section{REFERENCES}

I. Franchini M, Gandini G, Di Paolantonio T, et al. Acquired hemophilia A: a concise review. Am Hematol 2005;80(I):55-63.

2. Wendell CM, Bellazzini MA, Howes DS. Acquired factor VIII inhibitor presenting as a tongue hematoma. JEmerg Med 2004;26:4II-4.

3. Rinder H. Disorders of hemostasis: bleeding. In Andreoli TE, Carpenter CJ, Griggs RC, et al, editors. Cecil essentials of medicine. Philadelphia: WB Saunders; 200I. p. 457-72.

4. Mudad R, Kane WH. DDAVP in acquired hemophilia A: case report and review of the literature. Am J Hematol 1993;43:295-9.

5. Boggio LN, Green D. Acquired hemophilia. Rev Clin Exp Hematol 200I;5:389-404. 